\title{
Evidence for a neuroprotective microRNA pathway in amnestic mild cognitive impairment
}

\author{
Rebecca B. Weinberg ${ }^{1}$, Elliott J. Mufson ${ }^{2}$ and Scott E. Counts ${ }^{1,3,4 *}$ \\ ${ }^{1}$ Department of Translational Science and Molecular Medicine, Michigan State University, Grand Rapids, MI, USA, \\ ${ }^{2}$ Department of Neurobiology, Barrow Neurological Institute, Phoenix, AZ, USA, ${ }^{3}$ Department of Family Medicine, Michigan \\ State University, Grand Rapids, MI, USA, ${ }^{4}$ Hauenstein Neuroscience Center, Mercy Health Saint Mary's Hospital, Grand \\ Rapids, MI, USA
}

\section{OPEN ACCESS}

Edited by:

Raymond Scott Turner, Georgetown University, USA

Reviewed by: Jesus Avila

Centro de Biología Molecular Severo Ochoa CSIC-UAM, Spain

Julie A. Saugstad,

Oregon Health and Science University,

USA

Nandakumar Narayanan,

University of lowa, USA

*Correspondence:

Scott E. Counts

scott.counts@hc.msu.edu

Specialty section: This article was submitted to

Neurodegeneration, a section of the journa

Frontiers in Neuroscience

Received: 23 September 2015 Accepted: 22 October 2015 Published: 05 November 2015

Citation:

Weinberg RB, Mufson EJ and Counts SE (2015) Evidence for a neuroprotective microRNA pathway in amnestic mild cognitive impairment.

Front. Neurosci. 9:430

doi: 10.3389/fnins.2015.00430
MicroRNAs (miRNAs) that regulate mRNA stability have been linked to amyloid production, tau phosphorylation, and inflammation in Alzheimer's disease (AD). However, whether cerebral miRNA networks are dysregulated during the earliest stages of $A D$ remains underexplored. We performed miRNA expression analysis using frontal cortex tissue harvested from subjects who died with a clinical diagnosis of no cognitive impairment $(\mathrm{NCl})$, amnestic mild cognitive impairment (aMCl, a putative prodromal $A D$ stage), or mild AD. Analysis revealed that the miRNA clusters miR-212/132 and miR-23a/23b were down-regulated in the frontal cortex of aMCl subjects. Both miR-212/132 and miR23a/b are predicted to destabilize the message for sirtuin 1 (sirt1); hence, down-regulation of either miR-212/132 or miR-23a/b in frontal cortex should promote sirt1 mRNA expression in this region. qPCR studies revealed that frontal cortex levels of sirt1 were increased in aMCl. Given the ability of frontal cortex to respond to the onset of dementia by neuronal reorganization, these data suggest that miRNA-mediated up-regulation of the sirt1 pathway represents a compensatory response to the onset of the disease. By contrast, qPCR analysis of inferior temporal cortex, an area affected early in the progression of $A D$, showed no changes in miR-212/132, miR-23a/b, or sirt1 transcripts in the same aMCl subjects. In vitro mechanistic studies showed that coordinated down-regulation of miR-212 and miR-23a increased sirt1 protein expression and provided neuroprotection from $\beta$-amyloid toxicity in human neuronal cells. Taken together, these data suggest a novel miRNA-mediated neuroprotective pathway activated during the progression of $A D$ that may be amenable to therapeutic manipulation.

Keywords: Alzheimer's disease, mild cognitive impairment, frontal cortex, microRNAs, sirtuin 1, miR-23a, miR-212, gene expression regulation

\section{INTRODUCTION}

Progress in slowing the course of Alzheimer's disease (AD) has been confounded by a lack of disease modifying therapeutics. Given the vast complexity of this multisystem disorder, therapeutic development will likely depend on a deeper understanding of the intricate molecular mechanisms that regulate the maintenance and survival of selectively vulnerable neuronal populations during disease progression. In this regard, the presence of small non-coding microRNAs (miRNAs) 
that negatively regulate mRNA stability (Lagos-Quintana et al., 2001) presents an underexplored mechanism for fine-tuning gene expression within complex cellular networks, which likely plays a pivotal role in the balance between health and disease (Nelson et al., 2008; Hébert and De Strooper, 2009). Select miRNAs regulate diverse brain functions including neurogenesis and differentiation, synaptic plasticity, and energy metabolism (Schratt et al., 2006; Aschrafi et al., 2008; Fineberg et al., 2009; Rajasethupathy et al., 2009). This widespread influence of miRNA regulation on neuronal physiology suggests that perturbations in miRNA function could be involved in the pathogenesis of complex neurodegenerative disorders including $\mathrm{AD}$ (Nelson et al., 2008; Hébert and De Strooper, 2009). Indeed, AD brains display altered expression of several miRNAs that regulate $\beta$ secretase BACE1, a key enzyme involved in the generation of amyloid- $\beta$ (A $\beta$ ) plaque pathology (Hébert et al., 2008; Wang et al., 2008). In addition, miRNA dysregulation has been linked to tau phosphorylation (Hébert et al., 2010; Absalon et al., 2013; Banzhaf-Strathmann et al., 2014) and pro-inflammatory activity (Cui et al., 2010; Lukiw et al., 2010; Li et al., 2012). However, whether miRNA networks are dysregulated in the brains of people in the putative prodromal stages of $\mathrm{AD}$ such as amnestic mild cognitive impairment (aMCI) (Yaffe et al., 2006; Albert et al., 2011) and the extent to which these changes have physiologic consequences for the onset of $\mathrm{AD}$ remain unclear. To begin to address these knowledge gaps, we performed microarray and quantitative PCR (qPCR) studies to compare the levels of miRNAs isolated from frontal cortex (Brodmann area 10) and inferior temporal cortex (Brodmann area 20) tissue obtained postmortem from people who died with a clinical diagnosis of no cognitive impairment (NCI), aMCI, or $\mathrm{AD}$. We report that two families of miRNAs, miR-212/132 and miR-23a/b, were down-regulated in frontal cortex in aMCI and AD compared to NCI, yet remained stable in inferior temporal cortex. Downregulation of either miRNA family was predicted to up-regulate the deacetylase sirtuin 1 (sirt1), which is involved in mediating protective neuronal cell stress responses (Brunet et al., 2004; Qin et al., 2006; Bonda et al., 2011). Sirt1 mRNA levels were higher in frontal cortex of aMCI subjects but stable in inferior temporal cortex, suggesting a link between miR-212/132 and miR-23a/b down-regulation and reduced transcriptional repression of sirt1 target mRNA. Experimental down-regulation of miR-212 and miR-23a in cultured neurons up-regulated sirt 1 and provided neuroprotection against $A \beta$ toxicity. Given the relatively delayed involvement of frontal cortex in $\mathrm{AD}$ pathogenesis and the ability of this region to respond to the onset of dementia by neuronal reorganization (DeKosky et al., 2002; Counts et al., 2006; Bell et al., 2007; Williams et al., 2009; Bossers et al., 2010), these data suggest that miRNA-mediated up-regulation of sirt1 is a novel neuroprotective pathway activated during prodromal AD.

\section{MATERIALS AND METHODS}

\section{Subjects}

This study was exempt from IRB approval following guidelines for using de-identified postmortem tissue administrated by the
Rush University Medical Center. Frontal and inferior temporal cortex tissue was obtained postmortem from 32 participants in the Rush Religious Orders Study (Bennett et al., 2002) who were clinically diagnosed within a year of death with NCI $(n=12)$, aMCI $(n=10)$, or $\operatorname{AD}(n=10$; see Table 1). Details of clinical evaluations and diagnostic criteria have been previously published (Mufson et al., 1999; Counts et al., 2006; Ginsberg et al., 2010). In addition to an annual clinical evaluation, subjects were administered the Mini Mental State Exam (MMSE) and a battery of 19 neuropsychological tests referable to multiple cognitive domains (e.g., episodic memory, perceptual speed, Mufson et al., 1999). A Global Cognitive Score (GCS), consisting of a composite z-score calculated from this test battery, was determined for each participant (Bennett et al., 2002). The MCI population was defined as subjects who exhibited cognitive impairment on neuropsychological testing but who did not meet the clinical criteria for $\mathrm{AD}$ recommended by the joint working group of the National Institute of Neurologic and Communicative Disorders and Stroke/AD and Related Disorders Association (NINCDS/ADRDA) (McKhann et al., 1984; Bennett et al., 2002). The aMCI diagnosis is based on impairments in episodic memory (Yaffe et al., 2006; Albert et al., 2011). These criteria are compatible with those used by experts in the field to describe subjects who are not cognitively normal but do not meet established criteria for dementia (Petersen et al., 2001). "Other dementia" (OD) neurologic controls $(n=5)$ used in the analysis included three multi-infarct dementia and two Lewy body dementia cases.

Tissue samples were accrued as previously reported (Mufson et al., 1999; Counts et al., 2006; Ginsberg et al., 2010). At autopsy, tissue from one hemisphere was immersion-fixed in $4 \%$ paraformaldehyde in $0.1 \mathrm{M}$ phosphate buffer, $\mathrm{pH} 7.2$ for $24-72 \mathrm{~h}$ at $4^{\circ} \mathrm{C}$. Tissue slabs from the opposite hemisphere were frozen at $-80^{\circ} \mathrm{C}$ prior to biochemical analysis. Series of fixed tissue sections were prepared for neuropathological evaluation including visualization and quantitation of neocortical and hippocampal amyloid plaques and neurofibrillary tangles (NFTs) using antibodies directed against $A \beta$ peptide (A $\beta ; 4$ G8, Covance), tau (PHF1, a gift from Dr. Peter Davies) (Mufson et al., 1999; Bennett et al., 2002) as well as thioflavine-S histochemistry and a modified Bielschowsky silver stain. Additional sections were stained for Lewy bodies using antibodies directed against ubiquitin and $\alpha$-synuclein. Exclusion criteria included argyrophilic grain disease, frontotemporal dementia, Lewy body disease, mixed dementias, Parkinson's disease, and stroke. A board certified neuropathologist blinded to the clinical diagnosis performed the neuropathological evaluation. Neuropathological criteria were based on NIA-Reagan, CERAD, and Braak staging (Braak and Braak, 1991; Mirra et al., 1991; Hyman et al., 2012). Amyloid burden and apolipoprotein E (ApoE) genotype were determined for each case as described previously (Mufson et al., 1999; Bennett et al., 2002).

\section{miRNA Expression Profiling}

A pilot miRNA microarray screen (Exiqon miRCURY LNA microarray v.11, $\sim 1360$ array features including synthetic spikein miRNA controls) was performed using RNA derived from 
TABLE 1 | Clinical, demographic, and neuropathological characteristics by diagnosis category.

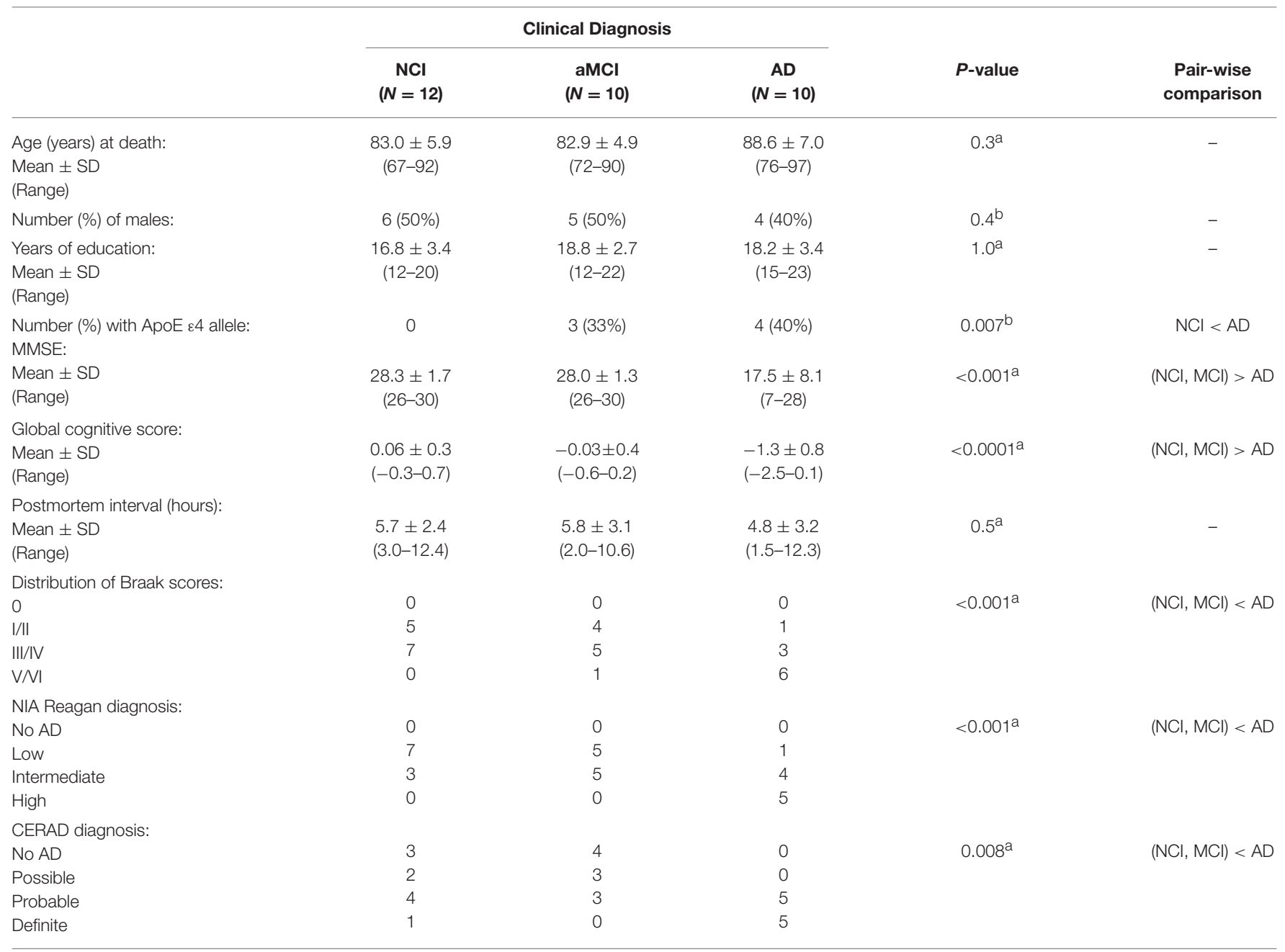

${ }^{a}$ Kruskal-Wallis test, with Bonferroni correction for multiple comparisons.

${ }^{b}$ Fisher's exact test, with Bonferroni correction for multiple comparisons.

postmortem frozen frontal cortex tissue from three NCI [age $=$ $86.7 \pm 1.5$ (mean \pm S.D.) years, MMSE $=29.7 \pm 0.6$, $\mathrm{PMI}=5.2 \pm 1.5 \mathrm{~h}]$ and three $\mathrm{AD}($ age $=87.7 \pm 1.5, \mathrm{MMSE}=$ $21.7 \pm 2.5, \mathrm{PMI}=5.2 \pm 1.7$ ) subjects. Approximately $30 \%$ of the miRNAs on the array were proprietary and not available for analysis in public databases. Based upon the microarray pilot data, we performed $\mathrm{qPCR}$ validation for select transcripts using frozen frontal cortex and temporal cortex from each of the NCI, aMCI, $\mathrm{AD}$, and $\mathrm{OD}$ neurologic control cases collected for the full study. Total RNA was extracted (miRvana, Ambion) and RNA integrity and concentration was verified using Bioanalysis (Agilent). Samples were assayed on a real-time PCR cycler (7900HT, Applied Biosystems) in 96-well optical plates as described previously (Counts et al., 2007; Ginsberg, 2008; Alldred et al., 2009). Target miRNAs of interest as well as the RNU48 artificial normalization control were amplified using specific Taqman hydrolysis probe sets (Applied Biosystems). In addition, Taqman probe sets specific for sirt1 and control glyceraldehyde 3-phosphate dehydrogenase were used to quantify sirt1 transcript levels in the same samples. The ddCT method was employed to determine relative expression levels of each amplicon (Counts et al., 2007; Ginsberg, 2008; Alldred et al., 2009). Variance component analyses revealed relatively low levels of within-case variability, and the average value of the triplicate qPCR products from each case was used in subsequent analyses.

\section{Dual in Situ \\ Hybridization/Immunohistochemical Localization of miR-23a and Sirt1}

In situ hybridization to detect miR-23a was performed on $10 \mu \mathrm{m}$, cryostat-sectioned samples of frozen frontal cortex using a digoxin (DIG)-labeled hsa-miR-23a probe (Exiqon), adapting the protocol of Doné and Beltcheva (2014). Briefly, tissue sections were fixed in $10 \%$ neutral buffered formalin overnight at room temperature (RT). The next day, sections were treated with $20 \mu \mathrm{g} / \mathrm{mL}$ proteinase $\mathrm{K}$ for $10 \mathrm{~min}$ at $37^{\circ} \mathrm{C}$ followed by hybridization with $400 \mathrm{nmol}$ hsa-miR-23a probe for $1 \mathrm{~h}$ at $55^{\circ} \mathrm{C}$. The sections were then blocked with $2 \%$ sheep serum $/ 1 \%$ bovine serum albumin for $15 \mathrm{~min}$ at RT 
followed by incubation with alkaline phosphatase-conjugated sheep anti-DIG Fab fragments (1:500, Roche) for $1 \mathrm{~h}$ at RT. The sections were then incubated with the alkaline phosphatase substrates NBT (nitro blue tetrazolium)/BCIP (5bromo-4-chloro-3-indolyl-phosphate; Roche) for $2 \mathrm{~h}$ at $30^{\circ} \mathrm{C}$ revealing a dark purple reaction product. Following miR-23a visualization, the sections were incubated overnight at $4^{\circ} \mathrm{C}$ with a rabbit anti-sirt1 monoclonal antibody (1:100, Origene) in Tris-buffered saline (TBS, $\mathrm{pH} 7.4$ )/0.25\% Triton X-100/1\% normal goat serum. Following TBS rinses, the sections were incubated with horseradish peroxidase-conjugated goat antirabbit secondary antiserum (Vector Laboratories) for $1 \mathrm{~h}$ at RT. Sirt1 labeling was accomplished by serial incubations in $\mathrm{ABC}$ peroxidase reagent (Vector Laboratories) and 3, 3 'diaminobenzidine tetrahydrochloride hydrate at RT to reveal a brown reaction product.

\section{Neuronal Cell Culture}

hNT neuronal cultures were derived from the human teratocarcinoma NT2 cell line (a gift from Dr. Virginia Lee, Univ. Penn) (Andrews et al., 1984; Counts and Mufson, 2010). NT2 cells were maintained in OptiMem (Invitrogen) with $5 \%$ fetal bovine serum (FBS). For differentiation, cells were seeded at $25,000 / \mathrm{cm}^{2}$ into T75 flasks in 1:1 DMEM/F-12 media (Invitrogen) $/ 10 \% \mathrm{FBS}$, treated twice a week with $10 \mu \mathrm{M}$ all-trans retinoic acid (Sigma) for 4 weeks and then seeded to new T75 flasks at $650,000 / \mathrm{cm}^{2}$ and treated with the mitotic inhibitors cytosine arabinoside $(1 \mu \mathrm{M})$ and fluorodeoxyuridine $(10 \mu \mathrm{M}$, Sigma) for 2 weeks. This resulted in a layer of phase-bright, post-mitotic neuronal cells loosely attached atop a monolayer of non-neuronal cells. Neuronal enrichment was achieved by gently trypsinizing the top neuronal layer and replating at $125,000 / \mathrm{cm}^{2}$ onto $2 \%$ Matrigel (BD Biosciences) and $10 \mu \mathrm{M}$ poly-D-lysine (Sigma)-coated black-walled 96 well plates (cell viability) or $60 \mathrm{~mm}$ dishes (western blotting) (BD Biosciences) in 1:1 DMEM/F-12 media/10\% FBS (Counts and Mufson, 2010).

\section{miRNA Inhibition and Functional Validation}

hNT cultures were transfected with small miRNA inhibitors (miRCURY LNA inhibitors, Exiqon) specific for miR-212, miR132, miR-23a, miR-23b, or an inhibitor negative control sequence (Exiqon) $(n=8 /$ treatment group in three independent experiments). hNT neurons were plated at $20 \mathrm{~K} / \mathrm{cm}^{2}$ and incubated with $50 \mathrm{nM}$ inhibitor/1\% Lipofectamine RNAiMAX (Life Technologies) in OptiMem for $18 \mathrm{~h}$, then exchanged into 1:1 DMEM/F-12 media/10\% FBS for $36 \mathrm{~h}$ prior to experimentation.

\section{Quantitative Western Blotting}

hNT neurons were harvested $36 \mathrm{~h}$ post-transfection and separated into nuclear and cytosolic fractions (NE-PER, Pierce) for quantitative immunoblotting. Nuclear proteins were solubilized in loading buffer and separated by SDS-PAGE, transferred to Immobilon-P membranes (Millipore), blocked in Tris buffered saline ( $\mathrm{pH}$ 7.4) containing 0.1\% Tween-20 and $2 \%$ nonfat milk, and then incubated overnight at $4{ }^{\circ} \mathrm{C}$ with rabbit polyclonal antiserum to sirt1 (1:500; Chemicon) and a mouse monoclonal antibody to lamin A (1:500, Abcam) as a loading control for the nuclear fraction. Blots were then incubated for $1 \mathrm{~h}$ with horseradish peroxidase-conjugated goat anti-rabbit (Bio-Rad; 1:5000) and anti-mouse (1:8000; Pierce, IL) IgG secondary antibodies and reactivity was quantified using Kodak 1D image analysis software (Perkin-Elmer). Each sample was analyzed on three different Western blots in independent experiments. Signals for sirt1 were normalized to lamin A for quantitative analysis (Counts et al., 2004, 2006).

\section{Amyloid Toxicity Experiments}

Following miRNA inhibitor or control transfections, differentiated hNT neurons were challenged with $10 \mu \mathrm{M}$ $\mathrm{A} \beta_{1-42}$ for $48 \mathrm{~h}$ in the presence or absence of the sirt1-specific inhibitor EX527 ( $100 \mathrm{nM}$, Tocris). $\mathrm{A} \beta_{1-42}$ was dissolved in DMSO and pre-aggregated for $16 \mathrm{~h}$ at $37^{\circ} \mathrm{C}$. Western blotting revealed an accumulation of SDS-soluble immunoreactive material migrating at $\sim 40-48 \mathrm{kDa}$ reminiscent of oligomeric amyloid (Walsh et al., 1999). Neuronal viability was determined by propidium iodide (PI) retention (Counts and Mufson, 2010).

\section{Statistical Analysis}

miRNA levels quantified by qPCR were compared among the $\mathrm{NCI}$, aMCI, $\mathrm{AD}$, and $\mathrm{OD}$ neurologic controls via One-way ANOVA with Bonferroni post-hoc testing. The relationship between specific miRNA and mRNA levels was assessed by Spearman rank correlations. Quantitative Western blotting data of control and miR-treated samples were analyzed by unpaired $t$ tests. Finally, cell death comparisons in the different cell culture treatment groups were assessed by One-way ANOVA with Bonferroni post-hoc testing. The level of statistical significance was set at $\alpha=0.05$ (two-tailed).

\section{RESULTS}

\section{Subject Demographics}

The clinical diagnostic groups did not differ by age, gender, years of education, or postmortem interval (Table 1). There were more subjects with an ApoE 4 allele in the aMCI (33\%) and AD (44\%) groups than in the NCI group $(0 \%)$. AD cases had significantly lower MMSE scores compared to both aMCI and NCI cases $(p<$ 0.001 ), whereas the latter two groups did not differ statistically (Table 1). GCS $z$-scores were significantly lower in AD compared to the NCI and aMCI groups $(p<0.0001)$. Subjects in the different clinical diagnostic groups displayed considerable heterogeneity with respect to pathological diagnostic criteria. Neuropathological examination revealed that $58 \%$ of NCI, $60 \%$ of aMCI, and $90 \%$ of AD cases were classified as Braak stages III-VI. Using NIA-Reagan criteria, 25\% of NCI, $50 \%$ of aMCI, and $90 \%$ of $\mathrm{AD}$ cases were classified as intermediate to high likelihood of AD (Table 1). For CERAD diagnosis, $42 \%$ of NCI, $30 \%$ of aMCI, and $100 \%$ of $\mathrm{AD}$ cases received a diagnosis of probable/definite $\mathrm{AD}$. Statistical analysis revealed that the $\mathrm{AD}$ group displayed significantly higher pathology than the NCI and aMCI groups based on Braak $(p<0.001)$, NIA-Reagan $(p<0.001)$ and CERAD $(p=0.008)$ diagnosis. 
TABLE 2 | Top 100 miRNAs dysregulated in AD compared to NCl frontal cortex.

\begin{tabular}{|c|c|c|c|c|c|}
\hline \multirow[t]{2}{*}{ Annotation } & \multirow[t]{2}{*}{$P$-value } & \multicolumn{2}{|c|}{ Average } & \multicolumn{2}{|c|}{ AD vs. $\mathrm{NCl}$} \\
\hline & & $\mathrm{NCl}$ & AD & $\Delta \mathrm{LMR}$ & Fold change \\
\hline hsa-miR-1285 & 5.92E-04 & 0.04 & -0.21 & -0.25 & 0.84 \\
\hline hsa-miR-1296 & $2.00 \mathrm{E}-03$ & -0.09 & -0.33 & -0.24 & 0.85 \\
\hline hsa-miR-668 & 5.48E-03 & -0.06 & -0.57 & -0.51 & 0.70 \\
\hline hsa-miR-551b* & 6.07E-03 & -0.45 & -0.01 & 0.44 & 1.36 \\
\hline hsa-miR-1826 & 6.49E-03 & -0.11 & -0.53 & -0.42 & 0.75 \\
\hline hsa-miR-518e* & 7.48E-03 & -0.44 & 0.15 & 0.59 & 1.50 \\
\hline hsa-miR-132 & 9.07E-03 & 0.53 & -0.22 & -0.74 & 0.60 \\
\hline hsa-miR-525-5p & 1.08E-02 & -0.09 & 0.15 & 0.24 & 1.18 \\
\hline hsa-miR-135b & 1.40E-02 & 0.45 & 0.17 & -0.29 & 0.82 \\
\hline hsa-miR-671-5p & 2.23E-02 & -0.24 & 0.00 & 0.24 & 1.18 \\
\hline hsa-miR-498 & 2.68E-02 & -0.23 & 0.29 & 0.51 & 1.43 \\
\hline hsa-miR-423-3p & 2.76E-02 & 0.05 & -0.17 & -0.22 & 0.86 \\
\hline hsa-miR-886-3p & 2.95E-02 & 0.32 & -1.62 & -1.94 & 0.26 \\
\hline hsa-miR-518a-5p & 3.76E-02 & -0.29 & 0.07 & 0.36 & 1.29 \\
\hline hsa-miR-204 & 3.97E-02 & 0.41 & 0.19 & -0.22 & 0.86 \\
\hline hsa-miR-886-5p & 4.21E-02 & 0.02 & -1.49 & -1.50 & 0.35 \\
\hline hsa-miR-132* & 4.25E-02 & 0.24 & -0.67 & -0.91 & 0.53 \\
\hline hsa-miR-129* & 4.38E-02 & 0.20 & -0.36 & -0.56 & 0.68 \\
\hline hsa-miR-382 & 4.08E-02 & 0.16 & -0.03 & -0.19 & 0.88 \\
\hline hsa-miR-921 & 5.35E-02 & -0.58 & -0.13 & 0.45 & 1.36 \\
\hline hsa-miR-140-5p & 5.67E-02 & 0.33 & -0.18 & -0.51 & 0.70 \\
\hline hsa-miR-330-5p & 5.87E-02 & 0.38 & 0.23 & -0.15 & 0.90 \\
\hline hsa-miR-510 & 6.01E-02 & -0.17 & 0.12 & 0.28 & 1.22 \\
\hline hsa-miR-491-3p & $6.15 \mathrm{E}-02$ & 0.23 & -0.35 & -0.57 & 0.67 \\
\hline hsa-miR-320a & 7.03E-02 & 0.29 & -0.27 & -0.56 & 0.68 \\
\hline hsa-miR-877 & 7.05E-02 & -0.11 & 0.17 & 0.28 & 1.22 \\
\hline hsa-miR-320b & 7.07E-02 & 0.29 & -0.26 & -0.55 & 0.68 \\
\hline hsa-miR-1252 & 7.13E-02 & -0.43 & 0.10 & 0.53 & 1.44 \\
\hline hsa-miR-30e & 7.40E-02 & 0.24 & -0.31 & -0.55 & 0.68 \\
\hline hsa-miR-129-3p & 7.40E-02 & 0.21 & -0.45 & -0.65 & 0.64 \\
\hline hsa-miR-149 & 7.43E-02 & 0.14 & -0.26 & -0.40 & 0.76 \\
\hline hsa-miR-25* & 7.49E-02 & -0.21 & 0.15 & 0.36 & 1.28 \\
\hline hsa-miR-149* & 7.57E-02 & -0.02 & 0.36 & 0.38 & 1.30 \\
\hline hsa-miR-483-5p & 7.63E-02 & -0.29 & 0.18 & 0.47 & 1.39 \\
\hline hsa-miR-630 & 7.94E-02 & -0.16 & 0.30 & 0.46 & 1.38 \\
\hline hsa-miR-1273 & 8.07E-02 & -0.45 & 0.14 & 0.59 & 1.50 \\
\hline hsa-miR-1264 & 8.34E-02 & 0.20 & -0.12 & -0.32 & 0.80 \\
\hline hsa-let-7a* & 8.46E-02 & 0.21 & -0.10 & -0.31 & 0.81 \\
\hline hsa-miR-505* & 8.53E-02 & -0.20 & 0.27 & 0.47 & 1.38 \\
\hline hsa-miR-377* & 8.62E-02 & 0.00 & 0.15 & 0.15 & 1.11 \\
\hline hsa-miR-21 & 8.77E-02 & 0.39 & -0.69 & -1.08 & 0.47 \\
\hline hsa-miR-126 & 8.81E-02 & 0.14 & -0.22 & -0.36 & 0.78 \\
\hline hsa-miR-625 & $9.22 \mathrm{E}-02$ & -0.20 & 0.11 & 0.31 & 1.24 \\
\hline hsa-miR-320d & 9.33E-02 & 0.17 & -0.21 & -0.39 & 0.77 \\
\hline hsa-miR-29b-2* & $9.40 \mathrm{E}-02$ & -0.01 & -0.33 & -0.33 & 0.80 \\
\hline hsa-miR-29c* & 9.41E-02 & 0.13 & -0.15 & -0.27 & 0.83 \\
\hline hsa-miR-30a & 9.58E-02 & 0.14 & -0.28 & -0.41 & 0.75 \\
\hline hsa-miR-1265 & 9.70E-02 & 0.20 & -0.03 & -0.23 & 0.85 \\
\hline
\end{tabular}

(Continued)
TABLE 2 | Continued

\begin{tabular}{|c|c|c|c|c|c|}
\hline \multirow[t]{2}{*}{ Annotation } & \multirow[t]{2}{*}{$P$-value } & \multicolumn{2}{|c|}{ Average } & \multicolumn{2}{|c|}{ AD vs. $\mathrm{NCl}$} \\
\hline & & $\mathrm{NCl}$ & AD & $\Delta$ LMR & Fold change \\
\hline hsa-miR-135a & $1.00 \mathrm{E}-01$ & 0.51 & -0.04 & -0.54 & 0.69 \\
\hline hsa-let-7i & 1.04E-01 & 0.15 & -0.33 & -0.48 & 0.72 \\
\hline hsa-miR-589 & 1.04E-01 & -0.14 & -0.46 & -0.32 & 0.80 \\
\hline hsa-miR-1201 & 1.06E-01 & -0.20 & -0.57 & -0.37 & 0.78 \\
\hline hsa-miR-1259 & 1.06E-01 & 0.12 & -0.22 & -0.34 & 0.79 \\
\hline hsa-miR-720 & 1.07E-01 & 0.18 & -0.24 & -0.42 & 0.75 \\
\hline hsa-miR-34b & 1.09E-01 & 0.10 & -0.21 & -0.32 & 0.80 \\
\hline hsa-let-7c & 1.09E-01 & 0.19 & -0.27 & -0.46 & 0.73 \\
\hline hsa-miR-320c & 1.09E-01 & 0.23 & -0.20 & -0.43 & 0.74 \\
\hline hsa-miR-583 & 1.12E-01 & -0.35 & 0.10 & 0.46 & 1.37 \\
\hline hsa-miR-206 & 1.12E-01 & -0.07 & 0.25 & 0.32 & 1.24 \\
\hline hsa-miR-23a & 1.12E-01 & 0.03 & -0.44 & -0.47 & 0.62 \\
\hline hsa-miR-181c & 1.12E-01 & -0.08 & -0.44 & -0.36 & 0.78 \\
\hline hsa-miR-769-5p & 1.16E-01 & 0.11 & -0.30 & -0.41 & 0.75 \\
\hline hsa-miR-181b & 1.17E-01 & 0.47 & -0.46 & -0.93 & 0.53 \\
\hline hsa-miR-1827 & 1.19E-01 & -0.26 & 0.29 & 0.55 & 1.46 \\
\hline hsa-miR-1253 & 1.20E-01 & -0.58 & -0.32 & 0.26 & 1.19 \\
\hline hsa-miR-301a & 1.20E-01 & 0.16 & -0.38 & -0.54 & 0.69 \\
\hline hsa-miR-184 & 1.21E-01 & -0.17 & 0.15 & 0.32 & 1.25 \\
\hline hsa-miR-140-3p & $1.21 \mathrm{E}-01$ & 0.14 & -0.46 & -0.61 & 0.66 \\
\hline hsa-miR-33a & 1.21E-01 & 0.31 & -0.27 & -0.58 & 0.67 \\
\hline hsa-miR-485-3p & 1.22E-01 & -0.18 & 0.16 & 0.34 & 1.26 \\
\hline hsa-miR-1274b & 1.22E-01 & 0.21 & -0.36 & -0.56 & 0.68 \\
\hline hsa-miR-181a* & $1.22 \mathrm{E}-01$ & 0.07 & -0.46 & -0.53 & 0.69 \\
\hline hsa-miR-516a-5p & 1.23E-01 & -0.42 & 0.02 & 0.44 & 1.36 \\
\hline hsa-miR-30d & 1.24E-01 & 0.24 & -0.11 & -0.35 & 0.78 \\
\hline hsa-miR-150 & 1.25E-01 & -0.28 & 0.38 & 0.66 & 1.58 \\
\hline hsa-miR-874 & $1.25 \mathrm{E}-01$ & -0.03 & -0.24 & -0.20 & 0.87 \\
\hline hsa-miR-339-5p & $1.27 \mathrm{E}-01$ & -0.03 & -0.48 & -0.45 & 0.73 \\
\hline hsa-miR-146b-5p & $1.28 \mathrm{E}-01$ & 0.29 & -0.26 & -0.55 & 0.68 \\
\hline hsa-let-7g & 1.28E-01 & 0.10 & -0.41 & -0.50 & 0.70 \\
\hline hsa-miR-142-3p & 1.28E-01 & 0.56 & 0.04 & -0.51 & 0.70 \\
\hline hsa-miR-212 & 1.29E-01 & -0.10 & -0.53 & -0.44 & 0.64 \\
\hline hsa-miR-518d-5p & 1.30E-01 & -0.12 & 0.08 & 0.19 & 1.14 \\
\hline hsa-miR-433 & 1.32E-01 & 0.03 & -0.14 & -0.18 & 0.89 \\
\hline hsa-miR-200b* & 1.34E-01 & -0.16 & 0.20 & 0.36 & 1.28 \\
\hline hsa-miR-491-5p & 1.34E-01 & 0.02 & -0.31 & -0.32 & 0.80 \\
\hline hsa-miR-24 & 1.35E-01 & 0.16 & -0.39 & -0.54 & 0.69 \\
\hline hsa-miR-193b* & 1.38E-01 & -0.26 & 0.19 & 0.46 & 1.37 \\
\hline hsa-miR-130b* & 1.38E-01 & -0.13 & 0.18 & 0.31 & 1.24 \\
\hline hsa-miR-30c & 1.40E-01 & 0.14 & -0.22 & -0.36 & 0.78 \\
\hline hsa-miR-23b & $1.41 \mathrm{E}-01$ & 0.13 & -0.53 & -0.66 & 0.63 \\
\hline hsa-miR-106b & $1.42 \mathrm{E}-01$ & 0.30 & -0.32 & -0.62 & 0.65 \\
\hline hsa-let-7b & 1.42E-01 & 0.30 & -0.14 & -0.45 & 0.73 \\
\hline hsa-let-7d & 1.43E-01 & 0.17 & -0.43 & -0.60 & 0.66 \\
\hline hsa-miR-647 & 1.43E-01 & -0.29 & 0.04 & 0.33 & 1.26 \\
\hline hsa-miR-181a & 1.43E-01 & 0.42 & -0.95 & -1.37 & 0.39 \\
\hline hsa-miR-130a & 1.44E-01 & 0.16 & -0.11 & -0.28 & 0.82 \\
\hline
\end{tabular}

${ }^{*}$ Star strand of miRNA. 


\section{miRNA Expression Dynamics during the Progression of AD}

A small pilot miRNA microarray screen performed on RNA isolated from frozen frontal cortex tissue from three NCI and $\mathrm{AD}$ cases showed that the expression levels of 30 miRNAs were significantly different between the two groups, along with miRNAs that showed high but non-significant magnitudes of expression change between the two groups. The top 100 miRNAs are listed in Table 2. We performed qPCR to measure select miRNAs in frontal cortex across all groups examined. Specifically, we tested the expression levels of 20 of the top 100 miRNAs based on significant differences, magnitude of expression level difference and/or interest based on a literature search (Cogswell et al., 2008; Hébert et al., 2013; Lau et al., 2013; Wong et al., 2013) (Table 3). Of the 20 candidate miRNAs evaluated, miR-498, and miR-150 were significantly upregulated in $\mathrm{AD}$ frontal cortex; miR-150 was up-regulated in aMCI. Several miRNAs were also significantly down-regulated in $\mathrm{AD}$ frontal cortex, including miR-886-3p, miR-132, miR21, miR-23a, miR-140-3p, miR-212, miR-23b, let-7d, and miR181a (Table 3). However, two distinct clusters of these miRNAs, $\mathrm{miR} 212 / 132$, and $\mathrm{miR} 23 \mathrm{a} / 23 \mathrm{~b}$, were also significantly downregulated by $\sim 50 \%$ in the frontal cortex of aMCI subjects relative to NCI subjects (Table 3, Figures 1A,B). By contrast, qPCR analysis of temporal cortex revealed that miR-212 and miR-132 expression levels were decreased only in the $\mathrm{AD}$ group, whereas miR-23a and miR-23b were unchanged across the clinical diagnostic groups (Table 4). Since miRNAs negatively regulate transcript stability, down-regulation of the

TABLE 3 | qPCR analysis of select miRNAs in frontal cortex of $\mathrm{NCl}, \mathrm{aMCl}$, and $A D$ subjects.

\begin{tabular}{|c|c|c|}
\hline miRNA & aMCl fold $\Delta$ vs. $\mathrm{NCl}$ & AD fold $\Delta$ vs. $\mathrm{NCl}$ \\
\hline hsa-miR-668 & 0.87 & 0.85 \\
\hline hsa-miR-135b & 0.76 & 0.84 \\
\hline hsa-miR-498 & 1.27 & $1.39^{*}$ \\
\hline hsa-miR-886-3p & 0.85 & $0.51^{* *}$ \\
\hline hsa-miR-132 & $0.58^{\star *}$ & $0.42^{\star \star}$ \\
\hline hsa-miR-320a & 0.98 & 0.91 \\
\hline hsa-miR-1252 & 1.17 & 1.14 \\
\hline hsa-miR-1273 & 1.03 & 1.22 \\
\hline hsa-miR-21 & 0.78 & $0.65^{\star}$ \\
\hline hsa-miR-23a & $0.53^{\star *}$ & $0.41^{* *}$ \\
\hline hsa-miR-181b & 0.88 & 0.93 \\
\hline hsa-miR-1827 & 1.27 & 1.21 \\
\hline hsa-miR-140-3p & 0.85 & $0.62^{*}$ \\
\hline hsa-miR-33a & 0.76 & 0.81 \\
\hline hsa-miR-150 & $1.43^{*}$ & $1.56^{\star \star}$ \\
\hline hsa-miR-212 & $0.47^{\star *}$ & $0.41^{\star *}$ \\
\hline hsa-miR-23b & $0.71^{*}$ & $0.62^{\star *}$ \\
\hline hsa-miR-106b & 1.05 & 0.97 \\
\hline hsa-let-7d & 0.73 & $0.66^{\star}$ \\
\hline hsa-miR-181a & 0.69 & $0.62^{*}$ \\
\hline
\end{tabular}

${ }^{*} p<0.05,{ }^{* *} p<0.01$
miR-212/132 and miR-23a/b clusters in frontal cortex would be predicted to promote the stability of their target mRNAs in aMCI. Given several lines of evidence that the frontal cortex undergoes neuroplastic, presumably neuroprotective remodeling in the face of mounting $\mathrm{AD}$ neuropathology during $\mathrm{MCI}$ (DeKosky et al., 2002; Counts et al., 2006; Bell et al., 2007; Williams et al., 2009; Bossers et al., 2010), we searched the miRanda and miRbase prediction algorithm databases (microRNA.org) for mRNA targets of these miRNAs that might mediate potentially compensatory pathways to promote neuronal viability. Surprisingly, all four miRNAs were predicted to target sirt1, a histone deacetylase involved in mediating neuronal cell stress responses (Brunet et al., 2004; Qin et al., 2006; Bonda et al., 2011). To determine whether target sirt1 transcripts were also differentially regulated in the frontal and temporal cortex in aMCI, we performed qPCR analysis of sirt1 expression levels in the same tissue samples used for miRNA analysis. This analysis revealed that sirt 1 mRNA levels were significantly upregulated by $\sim 40 \%$ in the frontal cortex of aMCI compared to NCI and AD subjects (Figure 1C). By contrast, sirt1 levels were stable in temporal cortex across the diagnostic groups (Table 4). Furthermore, increased sirt1 mRNA expression was significantly associated with decreased miR-212 levels frontal cortex levels, but this association was not found in the temporal cortex (Figure 2).

\section{Localization of miR-23a and Sirt1 in the Frontal Cortex}

Dual miR-23a in situ hybridization and sirt1 immunohistochemistry revealed nuclear miR-23a labeling in frontal cortex layer III neurons in tissue obtained from an 87 year-old female subject who died with a clinical diagnosis of aMCI (Figure 3). Sirt1 protein immunoreactivity was found primarily in the nucleus but also within the cytoplasm of the same neurons, providing evidence that miR-23a has direct access to its sirt 1 target and that miR-directed sirt1 regulation in cortex is neuronal in origin.

\section{miR-212 and miR-23a Down-regulation Increases Sirt1 Protein Expression in Human Neuronal Cells}

To determine the extent to which concomitant down-regulation of miR-212/132 and miR-23a/b and up-regulation of sirt1 observed in aMCI frontal cortex represented a functionally significant relationship, we treated human hNT neuronotypic cells with specific inhibitors of these miRNAs and measured sirt1 protein expression. Interestingly, inhibition of miR-212, miR132, miR-23a, or miR-23b individually had no effect on sirt1 expression, but we found that concurrent inhibition of miR212 and miR-23a resulted in a significant $\sim 100 \%$ increase in sirt1 (Figure 4), whereas co-inhibition of miR-132 and miR23a resulted in a $\sim 40 \%$ increase in sirt1 $(p<0.05$, data not shown). By contrast, miR-23b co-inhibition with miR212 or miR-132 had no effect on sirt1 expression (data not shown). 


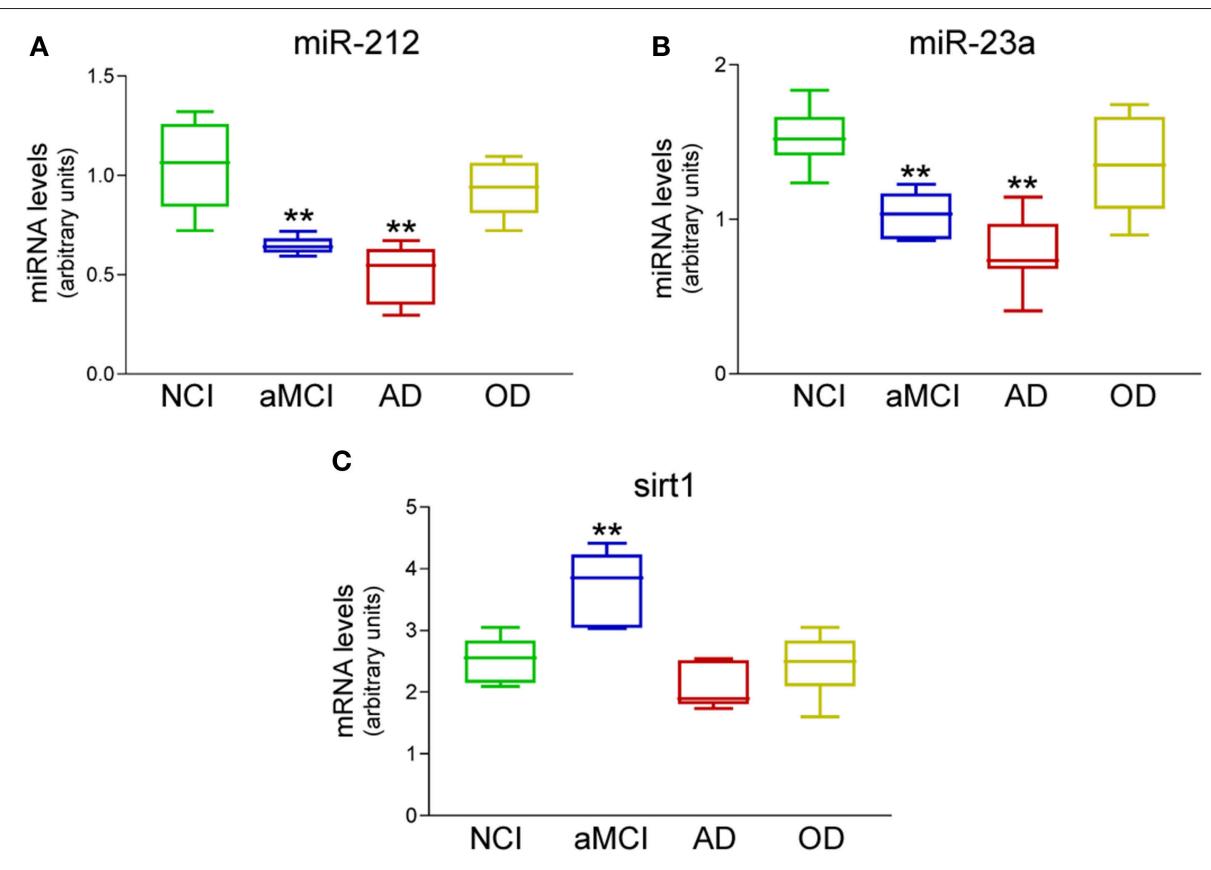

FIGURE 1 | Differential expression of miR-212, miR-23a, and sirt1 transcripts in the frontal cortex of aMCI subjects. qPCR analysis was performed on frozen frontal cortex tissue samples harvested from $\mathrm{NCI}(n=12)$, aMCI $(n=10)$, mild $\mathrm{AD}(n=10)$, and other dementia (OD, $n=5)$ neurologic control subjects. Box plots show that (A) miR-212 and (B) miR-23a were significantly down-regulated by $\sim 50 \%$ in aMCl and by $\sim 60 \%$ in AD. (C) Their predicted mRNA target sirt1 was up-regulated by $\sim 40 \%$ in aMCI. miR expression levels were normalized to the human RNU48 control miRNA, whereas sirt1 mRNA was normalized to GAPDH for quantitative analysis. ${ }^{*} p<0.01$ via One-way ANOVA with Bonferroni correction for multiple comparisons.

TABLE 4 | qPCR analysis of select miRNAs and sirt1 mRNA in temporal cortex of $\mathrm{NCl}$, aMCI, and $A D$ subjects.

\begin{tabular}{lcc}
\hline Probe set & aMCl fold $\boldsymbol{\Delta}$ vs. $\mathbf{N C l}$ & AD fold $\boldsymbol{\Delta}$ vs. $\mathbf{N C l}$ \\
\hline hsa-miR-212 & 0.96 & $0.63^{*}$ \\
hsa-miR-132 & 0.88 & $0.65^{*}$ \\
hsa-miR-23a & 0.92 & 0.87 \\
hsa-miR-23b & 1.03 & 0.98 \\
hsa-miR-150 & 1.32 & $1.47^{\star \star}$ \\
has-miR-668 & 0.95 & 1.01 \\
sirt 1 mRNA & 1.12 & 0.94
\end{tabular}

${ }^{*} p<0.05,{ }^{* *} p<0.01$.

\section{miR-23a and miR-212 Down-regulation Protects against $A \beta_{1-42}$ Induced Cell Death via Sirt1}

To test whether miR-212 and miR-23a regulation of sirt1 results in neuronal protection, hNT cells were treated with inhibitors of either miRNA independently, or with inhibitors of both miRNAs combined, followed by challenge with $A \beta_{1-42}$, which has been shown to induce cell death in these neuronal cells (Counts and Mufson, 2010). Inhibition of both miRNAs was sufficient to reduce $A \beta_{1-42}$ induced cell death (Figure 5). This response was blocked by co-administration with the sirt1 inhibitor EX527 (Figure 5), supporting the concept that miRNA regulation of sirt1 expression is a viable neuroprotective pathway.

\section{DISCUSSION}

The discovery of miRNAs (Lagos-Quintana et al., 2001) introduced a new layer of complexity to gene regulation, but also afforded the opportunity to better understand the molecular underpinnings of cellular function and dysfunction. This concept has already been well-demonstrated in cancer research and the potential therapeutic value of targeting miRNAs has been gaining acceptance in that field (Ma and Weinberg, 2008). With respect to neurodegenerative disorders such as $\mathrm{AD}$, the presence of miRNAs in the human brain and evidence for miRNA function in a wide variety of complex neuronal processes, including synaptic plasticity, suggests that miRNA regulation could have immense implications not only for disease pathogenesis but also for neuronal responses to progressive neurodegeneration (Kosik, 2006). Prior studies show that miRNAs could impact $\mathrm{AD}$ progression through several mechanisms. For instance, postmortem human tissue studies in well-characterized cohorts have shown decreased neocortical levels of miR-29a/b, miR9, and miR-107 in AD compared to control subjects, which was associated with increased BACE1 mRNA expression and A $\beta$ generation (Hébert et al., 2008; Wang et al., 2008; Che et al., 2014); in particular, miR-107 is downregulated very early in the disease process (Wang et al., 2008). By contrast, miR$15 \mathrm{a}$ is decreased in $\mathrm{AD}$ brain compared to healthy controls and is implicated in tau hyperphosphorylation in vivo (Hébert et al., 2010). Here, we show that mir-132/212 and miR$23 \mathrm{a} / \mathrm{b}$ are selectively down-regulated in the frontal cortex 

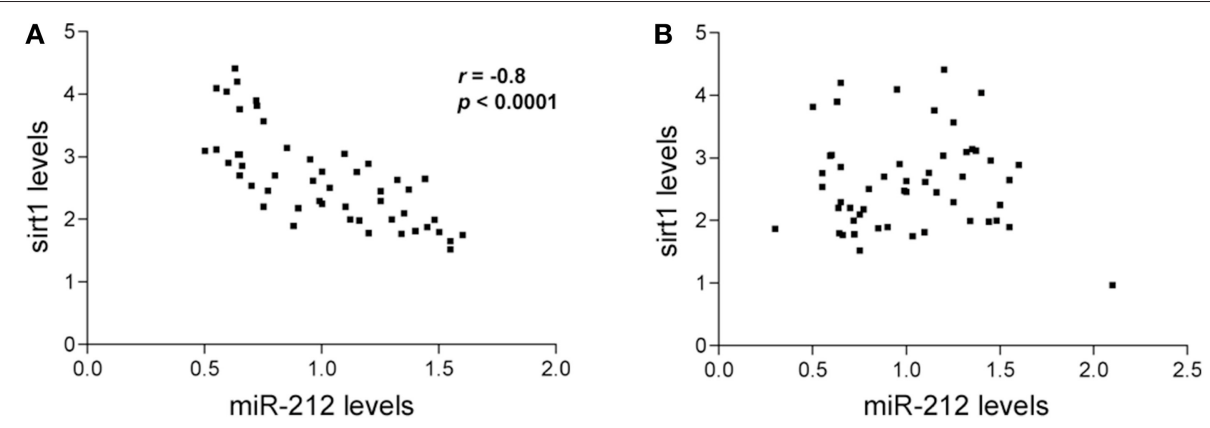

FIGURE 2 | Inverse relationship between miR-212 and sirt1 expression in the frontal cortex. Spearman rank correlations were performed to test for relationships between miR-212 and sirt1 expression. Scatterplots show that lower miR-212 transcript expression was associated with higher sirt1 transcript expression in (A) frontal cortex ( $r=-0.81, p<0.0001)$, but not in (B) temporal cortex in the subjects examined.

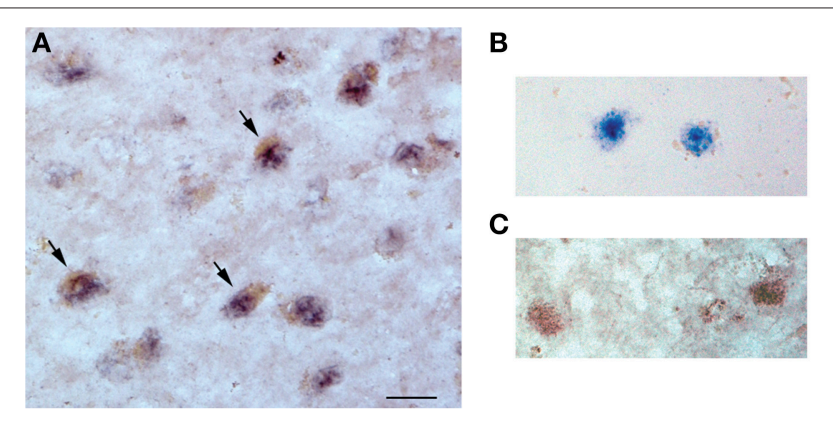

FIGURE 3 | miR-23a and sirt1 co-localize in the nucleus of frontal cortex layer III neurons. Dual in situ hybridization/immunohistochemistry of miR-23a RNA and sirt1 protein expression was performed in frontal cortex of an 87 year-old female subject who died with a clinical diagnosis of aMCl. (A) Photomicrograph shows layer III neurons co-labeled with miR-23a labeling (dark purple) and sirt1 (brown, arrows). Note that miR-23a appears localized to the nucleus, whereas sirt1 labeling is nuclear but also with immunoreactivity in the cytoplasm. (B) Dual miR-23a/sirt1 detection in the absence of sirt1 primary antibody (anti-rabbit lgG only). (C) Dual miR-23a/sirt1 detection with control scrambled miRNA probe. Scale bar $=30 \mu \mathrm{m}$.

in subjects clinically diagnosed with aMCI and that these alterations appear to be functionally linked to an up-regulation of sirt-1 and sirt-1 mediated protective responses. This novel finding adds to a growing literature on miRNA involvement in $\mathrm{AD}$ pathophysiology. However, rather than implicating another group of miRNAs in promoting neurodegeneration, our data support the concept that innate neuronal compensatory miRNA-mediated pathways are also activated in aMCI. A greater understanding of these and other miRNA pathways functioning during these putative prodromal stages of $\mathrm{AD}$ holds the promise that these pathways could be harnessed pharmacologically for drug development.

The miR-132/212 cluster has been implicated in several neuronal pathways, including dendritic elaboration (Magill et al., 2010) and learning and memory (Wang et al., 2013), and is downregulated in $\mathrm{AD}$ neocortex (Cogswell et al., 2008; Hébert et al., 2013; Lau et al., 2013; Wong et al., 2013). In particular, Wong and colleagues have previously shown that the
miR-212/132 cluster is down-regulated in temporal cortex in AD, and that inhibition of miR-212 and/or miR-132 expression can induce apoptosis in primary neurons after 1 week in culture via activation of a foxo3a-mediated cell death pathway (Wong et al., 2013). In this regard, we replicated the finding that the miR-212/132 cluster is down-regulated in the temporal cortex in $\mathrm{AD}$. However, we found no reductions in miR-212/132 in this region in aMCI; miR-212/132 was down-regulated in aMCI only in frontal cortex. In addition, in our hands experimental inhibition of miR-212 and/or miR-132 had no effect on cell survival of hNT neurons after $48 \mathrm{~h}$ in the absence of $\mathrm{A} \beta_{1-42}$. However, co-inhibition of either miR-212 or miR-132 with miR-23a conferred neuroprotection against $A \beta_{1-42}$ in a sirt1dependent manner. These discrepancies are likely explained by several factors. For instance, down-regulation of these miRNAs may have different functional implications in the AD temporal cortex, which displays a greater degree of degenerative changes than the aMCI frontal cortex. Notably, we found that sirtl upregulation in frontal cortex was confined to aMCI, suggesting that other AD-related pathways prevent sirt1 activation as the disease progresses. The down-regulation of miR-212/132 in aMCI frontal cortex may initially participate in protective compensatory mechanisms, but with sustained reductions these miRNAs may join a pathological cascade that promotes disease progression through foxo3a. In this regard, sirt1 activity has been shown to protect against foxo3a-mediated pro-apoptotic pathways by the deacetylation of this transcription factor (Brunet et al., 2004; Qin et al., 2008). Moreover, the differential roles for this cluster may depend on target binding partners, since miR-212/132 and miR-23a co-inhibition was required for sirt1 activation and neuroprotection. Hence, the activity of the miR$212 / 132$ cluster may be context dependent during the progression of $\mathrm{AD}$.

$\mathrm{miR}-23 \mathrm{a} / \mathrm{b}$ has also been shown to be dysregulated in the AD brain (Cogswell et al., 2008; Lau et al., 2013), yet much less is known about this miRNA cluster in neuronal function. However, like miR-212/132, miR-23a levels have been inversely linked to apoptosis. In a recent report detailing mechanisms for neuronal cell death in a model of traumatic brain injury, in vitro studies revealed that miR-23a inhibition 

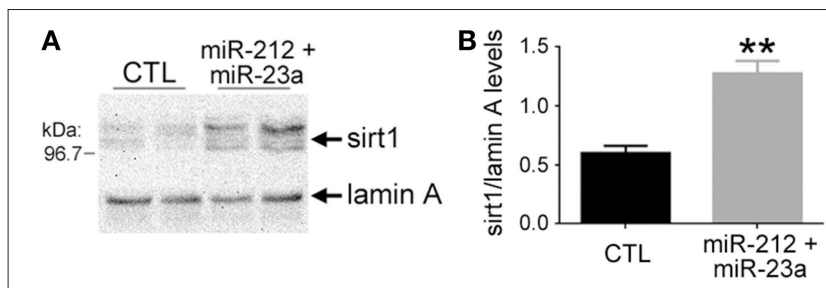

FIGURE 4 | Experimental down-regulation of miR-212 and miR-23a increases sirt1 protein expression. Human hNT neuronotypic cells were co-transfected with small inhibitors of miR-212 and 23a or scrambled negative controls (CTL). Given the co-localization of miR-23a and sirt1 in the nucleus, cells were harvested $36 \mathrm{~h}$ following transfection with the miRNA inhibitors or controls and nuclear fractions were immunoblotted for detection of sirt1. (A) A representative western shows that miR-212/23a co-repression resulted in an increase in sirt1 immunoreactivity. (B) Quantitative analysis revealed that sirt1 levels were significantly increased by $\sim 100 \%$ in miR-212/23a

inhibitor-transfected cells compared to CTL-transfected cells. $n=6 /$ group; ${ }^{\star *} p<0.01$ via Student's unpaired test (two-tailed).

increased etoposide-induced cell death after $24 \mathrm{~h}$ in cortical neurons via caspase activation (Sabirzhanov et al., 2014). By contrast, we found that miR-23a inhibition alone had no effect on $\mathrm{A} \beta$-induced cell death after $48 \mathrm{~h}$, yet was neuroprotective in the presence of miR-212 by activating sirt1. Again, these discordances are not incompatible, but suggest that miR-23a has both pro-apoptotic and neuroprotective properties that depend on specific miRNA binding partners and whether these miRNAs are targeting pro-apoptotic factors such as caspases or, as presently shown, factors such as sirtl that promote neuronal viability.

While the functional consequences of sirtl up-regulation in this paradigm are unclear, sirtl deacetylase activity plays an important role in regulating diverse cellular processes including aging, inflammation, and stress resistance (Imai et al., 2000; Brunet et al., 2004; North and Verdin, 2004; Herskovits and Guarente, 2014). In the adult brain, sirt1 can also modulate dendritic (Codocedo et al., 2012) and synaptic plasticity as well as memory formation (Gao et al., 2010; Michán et al., 2010). In addition to its importance during normal brain aging, sirt1 may also confer protective properties in neurodegenerative disorders such as AD (Qin et al., 2006, 2008; Min et al., 2010). Experimentally, sirt1 can reduce both $A \beta$-induced toxicity in neuronal cell lines (Conte et al., 2003; Han et al., 2004) and amyloid plaque formation in $\mathrm{AD}$ transgenic mice (Karuppagounder et al., 2009; Vingtdeux et al., 2010). Neuronal sirt1 expression has also been linked to non-amyloidogenic APP processing (Qin et al., 2006). Hence, we validated these concepts by showing that miR212/132 and miR-23a-mediated neuroprotection against $A \beta$ is prevented by a sirt1-specific inhibitor. Sirt1 has also been shown to ameliorate tangle-like pathology in tau mutant mice (Kim et al., 2007; Min et al., 2010), possibly by tau deacetylation that allows ubiquitin ligases to target tau for degradation (Min et al., 2010; Cohen et al., 2011). Hence, there are several mechanisms by which miR-212/132 and miR-23a co-regulation of sirt1 expression may promote neuroprotection in the frontal cortex in aMCI. However, it is

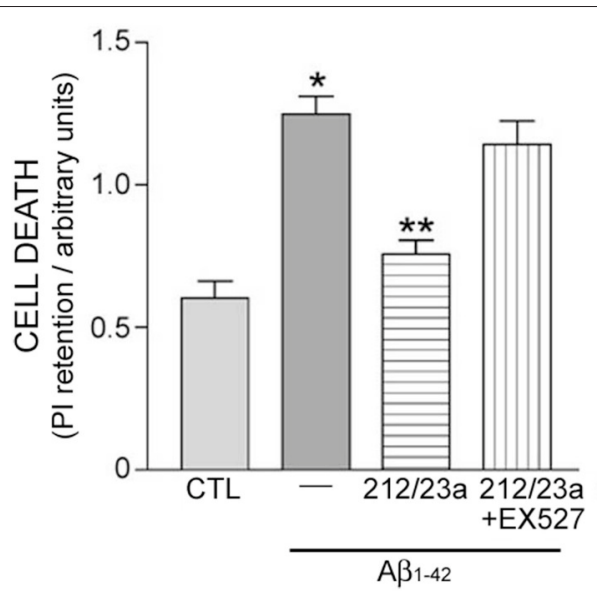

FIGURE 5 | Experimental down-regulation of miR-212 and miR-23a protects against $\mathbf{A} \beta_{1-42}$ in a sirt1-dependent manner. Human hNT neuronotypic cells were co-transfected with small inhibitors of miR-212 and 23a or scrambled negative controls (CTL). Cells were then treated with $10 \mu \mathrm{M}$ $\mathrm{A} \beta_{1-42}$ for $48 \mathrm{~h}$ in the presence or absence of $100 \mathrm{nM}$ EX527, a sirt1-specific inhibitor, followed by incubation with propidium iodide (PI) as an assay for cell death. Bar graph shows that $A \beta_{1-42}$ treatment resulted in significant cell death that was significantly reduced by miR-212/23a co-repression. This rescue effect was reversed by EX527, suggesting that the protective effects of miR-212/23a co-repression was mediated by sirt1. $n=8 /$ group; ${ }^{\star} p<0.05$ vs. $C T L ;{ }^{* *} p<0.05$ vs. $A \beta_{1-42}$ via One-way ANOVA with Bonferroni correction for multiple comparisons.

sirtl's role in the regulation and maintenance of dendritic growth (Codocedo et al., 2012) that could also play an important role in frontal cortex plasticity responses in MCI. In this regard, sirt1 knockout (KO) mice display reduced dendritic profiles relative to wild-type mice (Michán et al., 2010). Furthermore, overexpression of sirt1 can increase dendritic arborization, a phenotype that renders neurons resistant to dendritic dystrophy induced by $\mathrm{A} \beta$ (Codocedo et al., 2012). These data, taken together with multiple lines of evidence for a paradoxical up-regulation of synaptic elements within the frontal cortex during MCI (Counts et al., 2006; Bell et al., 2007; Williams et al., 2009; Bossers et al., 2010), suggest that sirt1 may mediate a synaptic remodeling response to mounting pathology during the progression of $\mathrm{AD}$.

In summary, our data suggest that the transition from normal cognitive function in aging to a clinical diagnosis of aMCI may involve the suppression of brain microRNA networks such as miR212/132 and miR-23a. In the frontal cortex, this may result in the induction of cellular survival pathways including the stabilization of sirt1. Ultimately, as aMCI progresses to $\mathrm{AD}$, the robust sirt1 expression is lost in frontal cortex, mimicking the situation seen in the temporal cortex. Recent microarray data suggest that the pattern of expression seen in sirt1 is also seen in other genes, particularly those involved in metabolic and synaptic machinery (Berchtold et al., 2013; Counts et al., 2013). Future research is necessary to confirm these findings in other $\mathrm{AD}$ cohorts, clarify whether the cumulative effect of these expression patterns is protective in vivo and by which sirt1-mediated mechanism, and determine whether augmentation of cell survival pathways such as sirt1 
represents a viable strategy to delay neurodegeneration in prodromal AD. Linking these concepts to recent advances in miRNA knockdown and mimetic technology will usher in a new class of powerful in vitro and in vivo approaches to test the efficacy of miRNA-based therapies in preclinical $\mathrm{AD}$ models.

\section{AUTHOR CONTRIBUTIONS}

RW participated in the experimentation and manuscript preparation. EM participated in the case selection, data analysis, and manuscript preparation. SC participated in the

\section{REFERENCES}

Absalon, S., Kochanek, D. M., Raghavan, V., and Krichevsky, A. M. (2013). MiR-26b, upregulated in Alzheimer's disease, activates cell cycle entry, tauphosphorylation, and apoptosis in postmitotic neurons. J. Neurosci. 33, 14645-14659. doi: 10.1523/JNEUROSCI.1327-13.2013

Albert, M. S., Dekosky, S. T., Dickson, D., Dubois, B., Feldman, H. H., Fox, N. C., et al. (2011). The diagnosis of mild cognitive impairment due to Alzheimer's disease: recommendations from the National Institute on AgingAlzheimer's Association workgroups on diagnostic guidelines for Alzheimer's disease. Alzheimers Dement. 7, 270-279. doi: 10.1016/j.jalz.2011.03.008

Alldred, M. J., Che, S., and Ginsberg, S. D. (2009). Terminal continuation (TC) RNA amplification without second strand synthesis. J. Neurosci. Methods 177, 381-385. doi: 10.1016/j.jneumeth.2008.10.027

Andrews, P. W., Damjanov, I., Simon, D., Banting, G. S., Carlin, C., Dracopoli, N. C., et al. (1984). Pluripotent embryonal carcinoma clones derived from the human teratocarcinoma cell line Tera-2. Differentiation in vivo and in vitro. Lab. Invest. 50, 147-162.

Aschrafi, A., Schwechter, A. D., Mameza, M. G., Natera-Naranjo, O., Gioio, A. E., and Kaplan, B. B. (2008). MicroRNA-338 regulates local cytochrome c oxidase IV mRNA levels and oxidative phosphorylation in the axons of sympathetic neurons. J. Neurosci. 28, 12581-12590. doi: 10.1523/JNEUROSCI.333808.2008

Banzhaf-Strathmann, J., Benito, E., May, S., Arzberger, T., Tahirovic, S., Kretzschmar, H., et al. (2014). MicroRNA-125b induces tau hyperphosphorylation and cognitive deficits in Alzheimer's disease. EMBO J. 33, 1667-1680. doi: 10.15252/embj.201387576

Bell, K. F., Bennett, D. A., and Cuello, A. C. (2007). Paradoxical upregulation of glutamatergic presynaptic boutons during mild cognitive impairment. J. Neurosci. 27, 10810-10817. doi: 10.1523/JNEUROSCI.3269-07.2007

Bennett, D. A., Wilson, R. S., Schneider, J. A., Evans, D. A., Beckett, L. A., Aggarwal, N. T., et al. (2002). Natural history of mild cognitive impairment in older persons. Neurology 59, 198-205. doi: 10.1212/WNL.59.2.198

Berchtold, N. C., Coleman, P. D., Cribbs, D. H., Rogers, J., Gillen, D. L., and Cotman, C. W. (2013). Synaptic genes are extensively downregulated across multiple brain regions in normal human aging and Alzheimer's disease. Neurobiol. Aging 34, 1653-1661. doi: 10.1016/j.neurobiolaging.2012.11.024

Bonda, D. J., Lee, H. G., Camins, A., Pallas, M., Casadesus, G., Smith, M. A., et al. (2011). The sirtuin pathway in ageing and Alzheimer disease: mechanistic and therapeutic considerations. Lancet Neurol. 10, 275-279. doi: 10.1016/S14744422(11)70013-8

Bossers, K., Wirz, K. T., Meerhoff, G. F., Essing, A. H., van Dongen, J. W., Houba, P., et al. (2010). Concerted changes in transcripts in the prefrontal cortex precede neuropathology in Alzheimer's disease. Brain 133, 3699-3723. doi: 10.1093/brain/awq258

Braak, H., and Braak, E. (1991). Neuropathological stageing of Alzheimer-related changes. Acta Neuropathol. 82, 239-259. doi: 10.1007/BF00308809

Brunet, A., Sweeney, L. B., Sturgill, J. F., Chua, K. F., Greer, P. L., Lin, Y., et al. (2004). Stress-dependent regulation of FOXO transcription factors by the SIRT1 deacetylase. Science 303, 2011-2015. doi: 10.1126/science.1094637 study design, experimentation, data analysis, and manuscript preparation.

\section{ACKNOWLEDGMENTS}

The authors would like to thank the participants of the Rush Religious Orders Study for their continued altruism and support of $\mathrm{AD}$ research. This work was supported by NIH AG042146, NIH AG014449, NIH AG043375, The Barrow Neurological Foundation and Barrow Beyond, and the Saint Mary's Foundation of Mercy Health Saint Mary's Hospital, Grand Rapids, MI.

Che, H., Sun, L. H., Guo, F., Niu, H. F., Su, X. L., Bao, Y. N., et al. (2014). Expression of amyloid-associated miRNAs in both the forebrain cortex and hippocampus of middle-aged rat. Cell. Physiol. Biochem. 33, 11-22. doi: 10.1159/0003 56646

Codocedo, J. F., Allard, C., Godoy, J. A., Varela-Nallar, L., and Inestrosa, N. C. (2012). SIRT1 regulates dendritic development in hippocampal neurons. PLoS ONE 7:e47073. doi: 10.1371/journal.pone.0047073

Cogswell, J. P., Ward, J., Taylor, I. A., Waters, M., Shi, Y., Cannon, B., et al. (2008). Identification of miRNA changes in Alzheimer's disease brain and CSF yields putative biomarkers and insights into disease pathways. J. Alzheimers Dis. 14, 27-41.

Cohen, T. J., Guo, J. L., Hurtado, D. E., Kwong, L. K., Mills, I. P., Trojanowski, J. Q., et al. (2011). The acetylation of tau inhibits its function and promotes pathological tau aggregation. Nat. Commun. 2, 252. doi: 10.1038/ncomm s1255

Conte, A., Pellegrini, S., and Tagliazucchi, D. (2003). Effect of resveratrol and catechin on PC12 tyrosine kinase activities and their synergistic protection from beta-amyloid toxicity. Drugs Exp. Clin. Res. 29, 243-255.

Counts, S. E., Alldred, M. J., Che, S., Ginsberg, S. D., and Mufson, E. J. (2013). Synaptic gene dysregulation within hippocampal CA1 pyramidal neurons in mild cognitive impairment. Neuropharmacology 79, 172-179. doi: 10.1016/j.neuropharm.2013.10.018

Counts, S. E., He, B., Che, S., Ikonomovic, M. D., DeKosky, S. T., Ginsberg, S. D., et al. (2007). Alpha7 nicotinic receptor up-regulation in cholinergic basal forebrain neurons in Alzheimer disease. Arch. Neurol. 64, 1771-1776. doi: 10.1001/archneur.64.12.1771

Counts, S. E., and Mufson, E. J. (2010). Noradrenaline activation of neurotrophic pathways protects against neuronal amyloid toxicity. J. Neurochem. 113, 649-660. doi: 10.1111/j.1471-4159.2010.06622.x

Counts, S. E., Nadeem, M., Lad, S. P., Wuu, J., and Mufson, E. J. (2006). Differential expression of synaptic proteins in the frontal and temporal cortex of elderly subjects with mild cognitive impairment. J. Neuropathol. Exp. Neurol. 65, 592-601. doi: 10.1097/00005072-200606000-00007

Counts, S. E., Nadeem, M., Wuu, J., Ginsberg, S. D., Saragovi, H. U., and Mufson, E. J. (2004). Reduction of cortical TrkA but not p75(NTR) protein in early-stage Alzheimer's disease. Ann. Neurol. 56, 520-531. doi: 10.1002/ana. 20233

Cui, J. G., Li, Y. Y., Zhao, Y., Bhattacharjee, S., and Lukiw, W. J. (2010). Differential regulation of interleukin-1 receptor-associated kinase-1 (IRAK1) and IRAK-2 by microRNA-146a and NF-kappaB in stressed human astroglial cells and in Alzheimer disease. J. Biol. Chem. 285, 38951-38960. doi: 10.1074/jbc.M110.178848

DeKosky, S. T., Ikonomovic, M. D., Styren, S. D., Beckett, L., Wisniewski, S., Bennett, D. A., et al. (2002). Upregulation of choline acetyltransferase activity in hippocampus and frontal cortex of elderly subjects with mild cognitive impairment. Ann. Neurol. 51, 145-155. doi: 10.1002/ana. 10069

Doné, S. C., and Beltcheva, O. (2014). In situ hybridization detection of miRNA using LNA oligonucleotides. Methods Mol. Biol. 1182, 57-71. doi: 10.1007/9781-4939-1062-5_6 
Fineberg, S. K., Kosik, K. S., and Davidson, B. L. (2009). MicroRNAs potentiate neural development. Neuron 64, 303-309. doi: 10.1016/j.neuron.2009.10.020

Gao, J., Wang, W. Y., Mao, Y. W., Gräff, J., Guan, J. S., Pan, L., et al. (2010). A novel pathway regulates memory and plasticity via SIRT1 and miR-134. Nature 466, 1105-1109. doi: 10.1038/nature09271

Ginsberg, S. D. (2008). Transcriptional profiling of small samples in the central nervous system. Methods Mol. Biol. 439, 147-158. doi: 10.1007/978-1-59745188-8_10

Ginsberg, S. D., Alldred, M. J., Counts, S. E., Cataldo, A. M., Neve, R. L., Jiang, Y., et al. (2010). Microarray analysis of hippocampal CA1 neurons implicates early endosomal dysfunction during Alzheimer's disease progression. Biol. Psychiatry 68, 885-893. doi: 10.1016/j.biopsych.2010.05.030

Han, Y. S., Zheng, W. H., Bastianetto, S., Chabot, J. G., and Quirion, R. (2004). Neuroprotective effects of resveratrol against beta-amyloid-induced neurotoxicity in rat hippocampal neurons: involvement of protein kinase C. Br. J. Pharmacol. 141, 997-1005. doi: 10.1038/sj.bjp.0705688

Hébert, S. S., and De Strooper, B. (2009). Alterations of the microRNA network cause neurodegenerative disease. Trends Neurosci. 32, 199-206. doi: 10.1016/j.tins.2008.12.003

Hébert, S. S., Horré, K., Nicolaï, L., Papadopoulou, A. S., Mandemakers, W., Silahtaroglu, A. N., et al. (2008). Loss of microRNA cluster miR-29a/b1 in sporadic Alzheimer's disease correlates with increased BACE1/betasecretase expression. Proc. Natl. Acad. Sci. U.S.A. 105, 6415-6420. doi: 10.1073/pnas.0710263105

Hébert, S. S., Papadopoulou, A. S., Smith, P., Galas, M. C., Planel, E., Silahtaroglu, A. N., et al. (2010). Genetic ablation of Dicer in adult forebrain neurons results in abnormal tau hyperphosphorylation and neurodegeneration. Hum. Mol. Genet. 19, 3959-3969. doi: 10.1093/hmg/ddq311

Hébert, S. S., Wang, W. X., Zhu, Q., and Nelson, P. T. (2013). A study of small RNAs from cerebral neocortex of pathology-verified Alzheimer's disease, dementia with lewy bodies, hippocampal sclerosis, frontotemporal lobar dementia, and non-demented human controls. J. Alzheimers Dis. 35, 335-348. doi: 10.3233/JAD-122350

Herskovits, A. Z., and Guarente, L. (2014). SIRT1 in neurodevelopment and brain senescence. Neuron 81, 471-483. doi: 10.1016/j.neuron.2014.01.028

Hyman, B. T., Phelps, C. H., Beach, T. G., Bigio, E. H., Cairns, N. J., Carrillo, M. C., et al. (2012). National Institute on Aging-Alzheimer's Association guidelines for the neuropathologic assessment of Alzheimer's disease. Alzheimers Dement. 8, 1-13. doi: 10.1016/j.jalz.2011.10.007

Imai, S., Armstrong, C. M., Kaeberlein, M., and Guarente, L. (2000). Transcriptional silencing and longevity protein Sir2 is an NAD-dependent histone deacetylase. Nature 403, 795-800. doi: 10.1038/35001622

Karuppagounder, S. S., Pinto, J. T., Xu, H., Chen, H. L., Beal, M. F., and Gibson, G. E. (2009). Dietary supplementation with resveratrol reduces plaque pathology in a transgenic model of Alzheimer's disease. Neurochem. Int. 54, 111-118. doi: 10.1016/j.neuint.2008.10.008

Kim, D., Nguyen, M. D., Dobbin, M. M., Fischer, A., Sananbenesi, F., Rodgers, J. T., et al. (2007). SIRT1 deacetylase protects against neurodegeneration in models for Alzheimer's disease and amyotrophic lateral sclerosis. EMBO J. 26, 3169-3179. doi: 10.1038/sj.emboj.7601758

Kosik, K. S. (2006). The neuronal microRNA system. Nat. Rev. Neurosci. 7, 911-920. doi: 10.1038/nrn2037

Lagos-Quintana, M., Rauhut, R., Lendeckel, W., and Tuschl, T. (2001). Identification of novel genes coding for small expressed RNAs. Science 294, 853-858. doi: 10.1126/science.1064921

Lau, P., Bossers, K., Janky, R., Salta, E., Frigerio, C. S., Barbash, S., et al. (2013). Alteration of the microRNA network during the progression of Alzheimer's disease. EMBO Mol. Med. 5, 1613-1634. doi: 10.1002/emmm.2012 01974

Li, Y. Y., Alexandrov, P. N., Pogue, A. I., Zhao, Y., Bhattacharjee, S., and Lukiw, W. J. (2012). miRNA-155 upregulation and complement factor $\mathrm{H}$ deficits in Down's syndrome. Neuroreport 23, 168-173. doi: 10.1097/WNR.0b013e32834 f4eb4

Lukiw, W. J., Cui, J. G., Yuan, L. Y., Bhattacharjee, P. S., Corkern, M., Clement, C., et al. (2010). Acyclovir or Abeta42 peptides attenuate HSV-1-induced miRNA-146a levels in human primary brain cells. Neuroreport 21, 922-927. doi: 10.1097/WNR.0b013e32833da51a
Ma, L., and Weinberg, R. A. (2008). Micromanagers of malignancy: role of microRNAs in regulating metastasis. Trends Genet. 24, 448-456. doi: 10.1016/j.tig.2008.06.004

Magill, S. T., Cambronne, X. A., Luikart, B. W., Lioy, D. T., Leighton, B. H., Westbrook, G. L., et al. (2010). microRNA-132 regulates dendritic growth and arborization of newborn neurons in the adult hippocampus. Proc. Natl. Acad. Sci. U.S.A. 107, 20382-20387. doi: 10.1073/pnas.1015691107

McKhann, G., Drachman, D., Folstein, M., Katzman, R., Price, D., and Stadlan, E. M. (1984). Clinical diagnosis of Alzheimer's disease: report of the NINCDSADRDA work group under the auspices of Department of Health and Human Services Task Force on Alzheimer's Disease. Neurology 34, 939-944. doi: 10.1212/WNL.34.7.939

Michán, S., Li, Y., Chou, M. M., Parrella, E., Ge, H., Long, J. M., et al. (2010). SIRT1 is essential for normal cognitive function and synaptic plasticity. J. Neurosci. 30, 9695-9707. doi: 10.1523/JNEUROSCI.0027-10.2010

Min, S. W., Cho, S. H., Zhou, Y., Schroeder, S., Haroutunian, V., Seeley, W. W., et al. (2010). Acetylation of tau inhibits its degradation and contributes to tauopathy. Neuron 67, 953-966. doi: 10.1016/j.neuron.2010.08.044

Mirra, S. S., Heyman, A., McKeel, D., Sumi, S. M., Crain, B. J., Brownlee, L. M., et al. (1991). The consortium to establish a registry for Alzheimer's Disease (CERAD). Part II. Standardization of the neuropathologic assessment of Alzheimer's disease. Neurology 41, 479-486. doi: 10.1212/WNL.41.4.479

Mufson, E. J., Chen, E. Y., Cochran, E. J., Beckett, L. A., Bennett, D. A., and Kordower, J. H. (1999). Entorhinal cortex beta-amyloid load in individuals with mild cognitive impairment. Exp. Neurol. 158, 469-490. doi: 10.1006/exnr.1999.7086

Nelson, P. T., Wang, W. X., and Rajeev, B. W. (2008). MicroRNAs (miRNAs) in neurodegenerative diseases. Brain Pathol. 18, 130-138. doi: 10.1111/j.17503639.2007.00120.x

North, B. J., and Verdin, E. (2004). Sirtuins: Sir2-related NAD-dependent protein deacetylases. Genome Biol. 5:224. doi: 10.1186/gb-2004-5-5-224

Petersen, R. C., Doody, R., Kurz, A., Mohs, R. C., Morris, J. C., Rabins, P. V., et al. (2001). Current concepts in mild cognitive impairment. Arch. Neurol. 58, 1985-1992. doi: 10.1001/archneur.58.12.1985

Qin, W., Yang, T., Ho, L., Zhao, Z., Wang, J., Chen, L., et al. (2006). Neuronal SIRT1 activation as a novel mechanism underlying the prevention of Alzheimer disease amyloid neuropathology by calorie restriction. J. Biol. Chem. 281, 21745-21754. doi: 10.1074/jbc.M602909200

Qin, W., Zhao, W., Ho, L., Wang, J., Walsh, K., Gandy, S., et al. (2008). Regulation of forkhead transcription factor FoxO3a contributes to calorie restrictioninduced prevention of Alzheimer's disease-type amyloid neuropathology and spatial memory deterioration. Ann. N.Y. Acad. Sci. 1147, 335-347. doi: 10.1196/annals. 1427.024

Rajasethupathy, P., Fiumara, F., Sheridan, R., Betel, D., Puthanveettil, S. V., Russo, J. J., et al. (2009). Characterization of small RNAs in Aplysia reveals a role for miR-124 in constraining synaptic plasticity through CREB. Neuron 63, 803-817. doi: 10.1016/j.neuron.2009.05.029

Sabirzhanov, B., Zhao, Z., Stoica, B. A., Loane, D. J., Wu, J., Borroto, C., et al. (2014). Downregulation of miR-23a and miR-27a following experimental traumatic brain injury induces neuronal cell death through activation of proapoptotic Bcl-2 proteins. J. Neurosci. 34, 10055-10071. doi: 10.1523/JNEUROSCI.1260-14.2014

Schratt, G. M., Tuebing, F., Nigh, E. A., Kane, C. G., Sabatini, M. E., Kiebler, M., et al. (2006). A brain-specific microRNA regulates dendritic spine development. Nature 439, 283-289. doi: 10.1038/nature04367

Vingtdeux, V., Giliberto, L., Zhao, H., Chandakkar, P., Wu, Q., Simon, J. E., et al. (2010). AMP-activated protein kinase signaling activation by resveratrol modulates amyloid-beta peptide metabolism. J. Biol. Chem. 285, 9100-9113. doi: $10.1074 /$ jbc.M109.060061

Walsh, D. M., Hartley, D. M., Kusumoto, Y., Fezoui, Y., Condron, M. M., Lomakin, A., et al. (1999). Amyloid beta-protein fibrillogenesis. Structure and biological activity of protofibrillar intermediates. J. Biol. Chem. 274, 25945-25952. doi: $10.1074 /$ jbc. 274.36 .25945

Wang, R. Y., Phang, R. Z., Hsu, P. H., Wang, W. H., Huang, H. T., and Liu, I. Y. (2013). In vivo knockdown of hippocampal miR-132 expression impairs memory acquisition of trace fear conditioning. Hippocampus 23, 625-633. doi: 10.1002/hipo.22123 
Wang, W. X., Rajeev, B. W., Stromberg, A. J., Ren, N., Tang, G., Huang, Q., et al. (2008). The expression of microRNA miR-107 decreases early in Alzheimer's disease and may accelerate disease progression through regulation of beta-site amyloid precursor protein-cleaving enzyme 1. J. Neurosci. 28, 1213-1223. doi: 10.1523/JNEUROSCI.506507.2008

Williams, C., Mehrian Shai, R., Wu, Y., Hsu, Y. H., Sitzer, T., Spann, B., et al. (2009). Transcriptome analysis of synaptoneurosomes identifies neuroplasticity genes overexpressed in incipient Alzheimer's disease. PLoS ONE 4:e4936. doi: 10.1371/journal.pone.0004936

Wong, H. K., Veremeyko, T., Patel, N., Lemere, C. A., Walsh, D. M., Esau, C., et al. (2013). De-repression of FOXO3a death axis by microRNA-132 and -212 causes neuronal apoptosis in Alzheimer's disease. Hum. Mol. Genet. 22, 3077-3092. doi: $10.1093 / \mathrm{hmg} / \mathrm{ddt} 164$
Yaffe, K., Petersen, R. C., Lindquist, K., Kramer, J., and Miller, B. (2006). Subtype of mild cognitive impairment and progression to dementia and death. Dement. Geriatr. Cogn. Disord. 22, 312-319. doi: 10.1159/000095427

Conflict of Interest Statement: The authors declare that the research was conducted in the absence of any commercial or financial relationships that could be construed as a potential conflict of interest.

Copyright (c) 2015 Weinberg, Mufson and Counts. This is an open-access article distributed under the terms of the Creative Commons Attribution License (CC BY).

The use, distribution or reproduction in other forums is permitted, provided the original author(s) or licensor are credited and that the original publication in this journal is cited, in accordance with accepted academic practice. No use, distribution or reproduction is permitted which does not comply with these terms. 